\title{
Elliptic functions and temperature inversion symmetry on spheres
}

\author{
J.S.Dowker ${ }^{1}$ \\ Department of Theoretical Physics, \\ The University of Manchester, \\ Manchester, England \\ and \\ Klaus Kirsten ${ }^{2}$ \\ Max Planck Institute for Mathematics in the Sciences, \\ Inselstrasse 22-26, 04103 Leipzig, Germany
}

\begin{abstract}
Finite temperature boson and fermion field theories on the space-time manifolds $\mathbb{R} \times \mathrm{S}^{d}$ are discussed with one eye on the questions of temperature inversion symmetry and modular invariance. For conformally invariant theories it is shown that the total energy at any temperature for any dimension, $d$, is given as a power series in the $d=3$ and $d=5$ energies, for scalars, and the $d=1$ and $d=3$ energies for spinors. Further, these energies can be given in finite terms at specific temperatures associated with singular moduli of elliptic function theory. Some examples are listed and numbers given.
\end{abstract}

\footnotetext{
1dowker@a35.ph.man.ac.uk

2 kirsten@mis.mpg.de
} 


\section{Introduction.}

The symmetry between low and high temperatures in finite temperature field theory has re-emerged recently in connection with the questions of entropy bounds and the Verlinde-Cardy relation. Some comments were made in an earlier paper [1] which we wish to enlarge on here. Although the main point of this earlier communication was the thermodynamic significance of any zero modes, this will occupy us only incidentally here.

The primary emphasis in the present article is on inversion symmetries in finite temperature field theory and its relation to elliptic functions. Inversion symmetry usually appears as a consequence of Jacobi's inversion identity for theta functions, in one of its many guises, and it should be no surprise that elliptic functions feature in our discussion.

In Section 2 we discuss for which manifolds simple inversion properties might be expected and we are naturally led to the manifold $S^{1} \times S^{d}, S^{1}$ being the thermal circle and $\mathrm{S}^{d}$ the $d$-dimensional sphere. A unified approach to the energy of quantum fields in curved space-time is via the thermal $\zeta$-function and some basics are presented in Section 3. Afterwards we relate the energy to Eisenstein series which allow symmetry properties to be discussed very easily. In particular, we can use relations between the Eisenstein series and the Weierstrass $\wp$-function to find the scalar energy for all dimensions just in terms of the results on the three and five sphere. For spinors one iterates from the one and three dimensional spheres. Elliptic functions are then used in Section 6 and 7 to give a description of temperature inversion in terms of the modulus, $k$. The relation to elliptic functions also allows one to express the energy in terms of powers of $k^{2}$ and of the complete elliptic integral, $K(k)$. This has the advantage that, at temperatures corresponding to singular moduli, closed forms for the energies can be found, see Section 9. The Conclusion describes the main points raised in the article. Three appendices contain technicalities about different viewpoints on the approaches for spinor fields, power series expansions of elliptic functions, and some explicit expressions too cumbersome for the main body of our paper.

\section{Background technical facts.}

Any symmetry relation has to be expressed in a dimensionless quantity say $a T=a / \beta \equiv \xi / 2 \pi$, where $a$ is some length scale associated with the spatial manifold, $\mathcal{M}$. Clearly reciprocal symmetry involves exchanging $\beta$ and $a$. 
Such a symmetry is therefore suggested whenever one can detect the torus structure, $\mathrm{S}^{1} \times \mathrm{S}^{1}$, in the thermal manifold, $\mathrm{S}^{1} \times \mathcal{M}$, the above exchange corresponding to a transposition of the two circles. An example where the symmetries are clearly visible is when $\mathcal{M}$ is the torus, $\mathrm{S}^{1} \times \mathrm{S}^{1} \times \ldots \times \mathrm{S}^{1}$. However the case that interests us here is when $\mathcal{M}$ is the $d$-sphere, in particular $d$ odd. The sphere, and all spaces of constant curvature, have only one relevant length scale, which at least simplifies the possibilities for reciprocation.

A basic analytical fact is that the scalar spectral quantities such as the heatkernel (or quantum propagator in our case) on the $d$-sphere can be 'integrated' in steps of two down to that on the circle, for theories conformal on the space-time. The thermal space, $\mathrm{S}^{1} \times \mathrm{S}^{d}$, reduces to $\mathrm{S}^{1} \times \mathrm{S}^{1}$. Alternatively, the thermal circle, $\mathrm{S}^{1}$, can be differentiated to give a "thermal $d$-sphere" resulting in the symmetrical combination $\mathrm{S}^{d} \times \mathrm{S}^{d}$. This occurs only if $d$ is odd and explains why inversion symmetries arise in this case. For even $d$, the scalar $\mathrm{S}^{1}$ heat-kernel can be fractionally differentiated to give $\mathrm{S}^{d}$, but only if it is twisted, i.e. a $\theta_{2}$-function, e.g. [2]. This is clearly the origin of Cardy's use of anti-periodic conditions for bosons, [3], for, in order to achieve any symmetry, the thermal circle must be twisted as well.

A practical strategy is to treat the terms in the degeneracy polynomial independently and deal with them one by one. Each part will have a different inversion property. It is this approach that will occupy us mostly in this paper.

We might mention that these calculations can be extended to split-rank symmetric spaces of which odd spheres are the simplest examples. (See, in this connection, [2].) The heat-kernel expansion terminates at the first term on all (semi)-simple group manifolds, but only if the scalar is conformal in 4 dimensions, whatever the dimension of the group.

\section{Statistical mechanics.}

The essential points were made in [1] but have to be repeated here for completeness and ease of exposition. A unified approach is via the thermal $\zeta$-function, $\zeta(s, \beta),[4-6]$, which neatly incorporates any ultra-violet and zero mode effects.

The general form of $\zeta(s, \beta)$ is, on an ultrastatic space-time, $\mathrm{T} \times \mathcal{M}$,

$$
\zeta(s, \beta)=\frac{i}{\beta} \sum_{\substack{m=-\infty \\ n}}^{\infty} \frac{d_{n}}{\left(\omega_{n}^{2}+4 \pi^{2} m^{2} / \beta^{2}\right)^{s}},
$$

where $\omega_{n}^{2}$ and $d_{n}$ are the eigenvalues and degeneracies of minus the Laplacian, $-\Delta_{2}$, 
on the spatial section, $\mathcal{M}$. The dash means that the denominator should never be zero.

The free energy (effective Lagrangian) is obtained from the limit

$$
F=\frac{i}{2} \lim _{s \rightarrow 0} \frac{\zeta(s, \beta)}{s}
$$

which, in general, can have infinities. Leaving these aside, in [1] the question of inversion symmetry was discussed directly, and most conveniently, from (1) as displaying most clearly any symmetry under interchange of $\beta$ and a scale $a$, which is embedded in the $\omega_{n}$, typically $\omega_{n} \sim 1 / a$.

It is possible to write the formalism in several equivalent forms often characterised by the way in which the mode information is organised. Each form has its own features. In [1] two such were outlined and it is the one which starts from the familiar statistical sum that we wish to concentrate on here.

In order to avoid certain problems, we deal only with internal energies, and leave for future work a treatment of the free energy and a complete thermodynamical scheme.

As shown in [4], the limit (2) leads, or can lead, to the expression for the total internal energy, $E=\partial(\beta F) / \partial \beta$,

$$
E=E_{0}+d_{0} T+\sum_{\omega_{n} \neq 0} \frac{d_{n} \omega_{n}}{e^{\beta \omega_{n}}-1}
$$

$E_{0}$ is the zero temperature value and may be the result of a regularisation/ renormalisation procedure. The effect of $d_{0}$ zero modes has also been included as the second term $[7,8]$. It is this form we wish to dwell on for a while as it allows one to make contact with some standard results in elliptic function theory.

\section{Scalars on the odd sphere.}

Information on modes on a sphere can be found in many places going back many years. For scalars conformal on the space-time, $\mathrm{T} \times \mathrm{S}^{d}$, the eigenvalues are perfect squares and the information can be displayed in the (zero temperature) $\zeta$-function,

$$
\zeta(s)=\frac{2}{(2 r) !} \sum_{n=1}^{\infty} \frac{\left(n^{2}-(r-1)^{2}\right) \ldots n^{2}}{\left(n^{2} / a^{2}\right)^{s}}, \quad d=2 r+1
$$


where, as mentioned in the Introduction, we are focusing on odd spheres. The degeneracy is a Gegenbauer polynomial. Another way of writing this involves the Barnes $\zeta$-function but we will not need this here.

Except for the circle, $d=1$, there are no zero modes so for simplicity let's restrict initially to $d>1$ and make the common expansion of the degeneracy (e.g. $[9])$,

$$
\left(n^{2}-(r-1)^{2}\right) \ldots n^{2}=\sum_{t=2}^{r+1}(-1)^{t+r} \mu_{t}(r) n^{2 t-2}
$$

which allows $\zeta(s)$ to be written as a sum of Riemann $\zeta$-functions, as has been done many times before. Our object is not to investigate this particular aspect much further but only to say that from this sum, or otherwise, it is possible to obtain the finite values of the zero temperature vacuum energy, $E_{0}$, as a sum of ordinary Bernoulli numbers, a calculation done a long time ago, and a number of times since. This is not the most compact form, but it is suitable for our purposes where, as has been indicated, we are going to treat the terms in (5) separately. For the $(2 t-2)$ th power we have

$$
a E_{0}(t)=\frac{1}{2} \zeta_{R}(1-2 t)=-\frac{B_{2 t}}{4 t}
$$

according to general theory $[4,10]$. (There is no need to use the Bernoulli number. One could just leave the Riemann $\zeta$-function, which is actually better.)

Let us therefore return to (3) and consider the 'partial' internal energies,

$$
a E(t)=-\frac{B_{2 t}}{4 t}+\sum_{n=1}^{\infty} \frac{n^{2 t-1}}{e^{2 \pi n / \xi}-1}
$$

and, in order to produce an expression that looks familiar, define a new parameter, $q$, by,

$$
q=e^{-\pi / \xi}
$$

to rewrite the internal energy (6) as a $q$-series,

$$
\epsilon_{t}(\xi) \equiv a E(t)=-\frac{B_{2 t}}{4 t}+\sum_{n=1}^{\infty} \frac{n^{2 t-1} q^{2 n}}{1-q^{2 n}}
$$

The summation is an example of a Lambert series and occurs in analytic number and in elliptic function theory. In the former, $q$ usually is the square of the $q$ here, (7). 
The most rapid way of proceeding is to note the standard result that the combination (8) is, up to a factor, an Eisenstein series. Precisely,

$$
\epsilon_{t}(\xi)=(-1)^{t} \frac{(2 t-1) !}{2(2 \pi)^{2 t}} G_{t}(1, i / \xi),
$$

where the Eisenstein series is defined by (we are following Hurwitz, [11,12], here),

$$
G_{t}\left(\omega_{1}, \omega_{2}\right)=\sum_{\substack{m_{1}, m_{2} \\=-\infty}}^{\infty} \frac{1}{\left(m_{1} \omega_{1}+m_{2} \omega_{2}\right)^{2 t}} .
$$

When $t=1$, the torus case, the sums have to be considered more carefully. This point will be returned to. A useful discussion is given by Rademacher [13].

The behaviour of $\epsilon_{t}(\xi)$ under inversion is now apparent from (9) and the structure of the Eisenstein series (10), i.e.

$$
\epsilon_{t}(1 / \xi)=(-1)^{t} \frac{1}{\xi^{2 t}} \epsilon_{t}(\xi) .
$$

The $G_{k}$ are holomorphic modular forms, invariant under $\mathrm{SL}(2, \mathbb{Z})$ action on the periods $\omega_{1}, \omega_{2}$. The simple inversion case $\xi \rightarrow 1 / \xi$ produces (11). The more general action contains other information.

The result (11) when re-expressed in terms of the expression in (8) is equivalent to the duality relation,

$$
\mu^{t} \sum_{n=1}^{\infty} \frac{n^{2 t-1}}{e^{2 \mu n}-1}-\left(-\mu^{\prime}\right)^{t} \sum_{n=1}^{\infty} \frac{n^{2 t-1}}{e^{2 \mu^{\prime} n}-1}=\left(\mu^{t}-\left(-\mu^{\prime}\right)^{t}\right) \frac{B_{2 t}}{4 t}, \quad \mu \mu^{\prime}=\pi^{2},
$$

which is given by Ramanujan, typically without proof. It was derived later by Rao and Ayyar, [14] and thereafter by many workers including Malurkar [15] and Hardy [16] who use Mellin transforms. Berndt [17] gives some history which we could modify by saying that, since (12) follows immediately from (9), then it is contained in Glaisher, [18] and, by the same token, appears even earlier in Hurwitz [11,12].

The Eisenstein series enter into the Laurent expansion of the Weierstrass $\wp-$ function with periods $\omega_{1}$ and $\omega_{2}$,

$$
\wp(u)=\frac{1}{u^{2}}+\sum_{t=2}^{\infty}(2 t-1) G_{t} u^{2 t-2},
$$

which can be regarded as a generating function for the internal energies, $\epsilon_{t}$. 
Because of the restrictions of analyticity and periodicity, $\wp$ satisfies the Weierstrass differential equation, (originally obtained by Eisenstein),

$$
\begin{aligned}
\wp^{\prime}(u)^{2} & =4 \wp(u)^{3}-60 G_{2}\left(\omega_{1}, \omega_{2}\right) \wp(u)-140 G_{3}\left(\omega_{1}, \omega_{2}\right) \\
& \equiv 4 \wp(u)^{3}-g_{2}\left(\omega_{1}, \omega_{2}\right) \wp(u)-g_{3}\left(\omega_{1}, \omega_{2}\right)
\end{aligned}
$$

which leads to a recursion formula giving all the $G_{t}$ as algebraic polynomials in just the two invariants, $g_{2}$ and $g_{3}$, with rational coefficients, which is a very remarkable fact. A few examples are given in [12], footnote on p.33 and a larger list in [19] IV p.88.

The calculation has been kindly done for us by Rademacher, [13], and gives, expressed in the $\epsilon_{t}$,

$$
\epsilon_{t}(\xi)=12 \frac{(t-1)(2 t-3)}{(2 t+1)(t-3)} \sum_{l=2}^{t-2}\left(\begin{array}{l}
2 t-4 \\
2 l-2
\end{array}\right) \epsilon_{l}(\xi) \epsilon_{t-l}(\xi), \quad t \geq 4
$$

This is an identity in $\xi$ and yields several specific identities generally of arithmetic interest. For example, in the low temperature limit, $\xi=0$, i.e. $q=0$, (14) reduces to an identity between Bernoulli numbers, e.g. [13] p.124.

The recursion is more simply expressed in terms of the expansion coefficients, $T_{t} \equiv(2 t-1) G_{t}$, in (13), e.g. [20] p.92, [19] Vol I, p.176, [21] p.155, [22] p.11, as

$$
T_{t}=\frac{3}{(t-3)(2 t+1)} \sum_{l=2}^{t-2} T_{l} T_{t-l}
$$

Examples of the minimal polynomials resulting from (14) are

$$
\begin{gathered}
\epsilon_{4}=120 \epsilon_{2}^{2}, \quad \epsilon_{5}=\frac{2^{4} 3^{2} 57}{11} \epsilon_{2} \epsilon_{3}, \quad \epsilon_{6}=\frac{2^{3} 3^{2} 5^{2} 7}{13}\left(96 \epsilon_{2}^{3}+\epsilon_{3}^{2}\right), \\
\epsilon_{7}=2^{8} 3^{3} 5^{2} 7 \epsilon_{2}^{2} \epsilon_{3}, \quad \epsilon_{8}=\frac{2^{8} 3^{4} 5^{3} 7^{2}}{17} \epsilon_{2}\left(44 \epsilon_{2}^{3}+\epsilon_{3}^{2}\right) .
\end{gathered}
$$

The total energy on the $d$-sphere is, according to equations (3), (5) and (6), a sum of the $\epsilon_{t}$,

$$
a E_{\mathrm{S}^{d}} \equiv \bar{E}_{d}(\xi)=\frac{2}{(2 r) !} \sum_{t=1}^{r}(-1)^{t+r} \mu_{t}(r) \epsilon_{t+1}(\xi), \quad d=2 r+1
$$

and therefore can also be expressed as a polynomial in $\epsilon_{2}$ and $\epsilon_{3}$, or, equivalently in the three- and five-sphere energies, $\bar{E}_{3}$ and $\bar{E}_{5}$. 
This has numerical import since it means that the energies on all $d$-spheres are known once those on $S^{3}$ and $S^{5}$ are and this is true for all temperatures, including zero. For example, the energies up to the nine-sphere are,

$$
\begin{aligned}
& \bar{E}_{3}=\epsilon_{2} \\
& \bar{E}_{5}=\frac{1}{12}\left(\epsilon_{3}-\epsilon_{2}\right) \\
& \bar{E}_{7}=\frac{1}{360}\left(120 \epsilon_{2}^{2}-5 \epsilon_{3}+4 \epsilon_{2}\right) \\
& \bar{E}_{9}=\frac{1}{221760}\left(5040 \epsilon_{2} \epsilon_{3}-18480 \epsilon_{2}^{2}+539 \epsilon_{3}-396 \epsilon_{2}\right) .
\end{aligned}
$$

Apart from this, the behaviour of the energy on $\mathrm{S}^{d}$ under the inversion $\xi \rightarrow$ $1 / \xi$ is determined from (17) and the individual behaviours, (11). In this way we regain the high temperature form derived generally in [4] in terms of the heat-kernel coefficients. It is another way of introducing, organising and using the spectral information.

Quantities equivalent to the $\epsilon_{t}$ are considered by Ramanujan [23] who computes the expressions analogous to (16) by a recursion derived purely algebraically.

\section{Spinors on the odd sphere.}

The spinor spectral data on the sphere are well known so we can be brief and simply display them via the zero-temperature $\zeta$-function on odd spheres,

$$
\zeta(s)=\frac{2^{(d+1) / 2}(-1)^{(d-1) / 2}}{(d-1) !} \sum_{t=0}^{(d-1) / 2} \sum_{n=0}^{\infty} \frac{\nu_{t}(n+1 / 2)^{2 t}}{\left((n+1 / 2)^{2} / a^{2}\right)^{s}}
$$

where the degeneracy has again been expanded and some re-arrangement of the starting points made for convenience.

We will again treat the individual powers in the degeneracy separately, and, putting some constants aside for ease, at finite temperature the fermion partial internal energies are defined by,

$$
\eta_{t}(\xi)=-\frac{1}{2} \zeta_{t}(-1 / 2)+\sum_{n=0}^{\infty} \frac{(2 n+1)^{2 t-1}}{e^{\pi(2 n+1) / \xi}+1},
$$

where the first term is the zero temperature part with,

$$
\zeta_{t}(s)=2^{2 t-2 s-2} \zeta_{R}(2 s-2 t+2,1 / 2)
$$


which can be given in terms of Bernoulli numbers, if desired, as

$$
-\frac{1}{2} 2^{2 t-1} \zeta_{R}(1-2 t, 1 / 2)=\left(1-2^{2 t-1}\right) \frac{B_{2 t}}{4 t}=2^{2 t-1} \frac{B_{2 t}(1 / 2)}{4 t} .
$$

The point in writing it in this particular way is that we can now immediately appeal to a formula given by Glaisher, [18] p.64, which reads, after transcribing the notation and reverting to the double-sided summations,

$$
\begin{aligned}
\sum_{n=0}^{\infty} \frac{(2 n+1)^{2 t-1} q^{2 n+1}}{1+q^{2 n+1}}=-(1- & \left.2^{2 t-1}\right) \frac{B_{2 t}}{4 t}+ \\
& \frac{(-1)^{t+1}(2 t-1) !}{4 \pi^{2 t}} \sum_{\substack{m_{1}, m_{2} \\
=-\infty}}^{\infty} \frac{(-1)^{m_{1}+m_{2}}}{\left(m_{1}+i m_{2} / \xi\right)^{2 t}}
\end{aligned}
$$

where $q=e^{-\pi / \xi}$. A glance at (20), (21) and (22) shows that the spinor partial energy is given by a sign-modulated, doubly twisted Eisenstein series, $H_{t}$,

$$
\eta_{t}(\xi)=\frac{(-1)^{t+1}(2 t-1) !}{4 \pi^{2 t}} H_{t}(1, i / \xi)
$$

where,

$$
H_{t}\left(\omega_{1}, \omega_{2}\right)=\sum_{\substack{m_{1}, m_{2} \\=-\infty}}^{\infty} \frac{(-1)^{m_{1}+m_{2}}}{\left(m_{1} \omega_{1}+m_{2} \omega_{2}\right)^{2 t}}
$$

and obviously, therefore, enjoys the same inversion properties as the scalar quantity, $\epsilon_{t}(\xi)$, i.e.

$$
\eta_{t}(1 / \xi)=(-1)^{t} \frac{1}{\xi^{2 t}} \eta_{t}(\xi)
$$

Re-expressed by (20), this result becomes,

$$
\mu^{t} \sum_{n=0}^{\infty} \frac{(2 n+1)^{2 t-1}}{e^{(2 n+1) \mu}+1}-\left(-\mu^{\prime}\right)^{t} \sum_{n=0}^{\infty} \frac{(2 n+1)^{2 t-1}}{e^{(2 n+1) \mu^{\prime}+1}}=-\left(\mu^{t}-\left(-\mu^{\prime}\right)^{t}\right)\left(1-2^{2 t-1}\right) \frac{B_{2 t}}{4 t}
$$

for $\mu \mu^{\prime}=\pi^{2}$. According to Berndt, [24], this formula is due to Rao and Ayyar, [14], but we see that it is contained in Glaisher, [18] (but not in Hurwitz [11]).

Without going into details, we note at this point that relations more general than (12) and (26) hold when $t$ is allowed to be negative.

As an already discussed example, [25], take the three-sphere. The total internal spinor energy is,

$$
\bar{E}_{3}^{\mathrm{f}}(\xi)=\frac{1}{2}\left(\eta_{2}(\xi)-\eta_{1}(\xi)\right)
$$


Under inversion,

$$
\bar{E}_{3}^{\mathrm{f}}(1 / \xi)=\frac{1}{2}\left(\frac{1}{\xi^{4}} \eta_{2}(\xi)+\frac{1}{\xi^{2}} \eta_{1}(\xi)\right) .
$$

In this case the heat-kernel expansion terminates at the second term.

The twisted Eisenstein series, $H_{t},(24)$, appear as coefficients in the Laurent series for the twisted Weierstrass function, $\widetilde{\wp}$, (an 'elliptic function of the second kind') defined by,

$$
\widetilde{\wp}(u)=\frac{1}{u^{2}}+\sum_{\substack{m_{1}, m_{2} \\=-\infty}}^{\infty}\left(\frac{(-1)^{m_{1}+m_{2}}}{\left(u-m_{1} \omega_{1}-m_{2} \omega_{2}\right)^{2}}-\frac{(-1)^{m_{1}+m_{2}}}{\left(m_{1} \omega_{1}+m_{2} \omega_{2}\right)^{2}}\right) .
$$

This will appear later in Appendices A and B.

However, in order to derive fermion equations analogous to the recursive (18), it seems that Jacobi functions are more suitable and so we turn to this description.

\section{Elliptic functions and alternative descriptions.}

In this, and the following section we expand on the previous analysis and also discuss inversion relations using different ingredients.

Hurwitz proves (9) directly, starting from the partial fraction expansion for the cotangent. The proof in Rademacher [13] involves a slight reorganisation and an application of Lipschitz' formula,

$$
\frac{(2 \pi)^{s}}{\Gamma(s)} \sum_{m=0}^{\infty}(m+\alpha)^{s-1} e^{-2 \pi z(m+\alpha)}=\sum_{n=-\infty}^{\infty} \frac{e^{2 \pi i n \alpha}}{(z+n i)^{s}},
$$

to the $m_{1}$ summation. Equation (28) is a duality relation and can be proved using Poisson summation. There are some restrictions. Thus $\operatorname{Re} z>0,0<\alpha \leq 1$ and $\operatorname{Re} s>1$. If $0<\alpha<1$ then $\operatorname{Re} s>0$.

The result, (9), is also given in Glaisher, [18] §72, and Halphen, [20], proved in slightly different manners. Halphen gives some specific examples on p.446. (There is a minor error of 4 in the third line of his eqn.(78).) See also Hurwitz [12] pp.22,33.

Halphen employs Weierstrass' $\wp$, whereas Glaisher develops everything from the $q$-series for Jacobi elliptic functions. The ones relevant here for bosons and fermions are those for zs and ds, the former being Glaisher's notation for the Jacobi $\zeta$-function, $Z_{1}$. The following trigonometric expansions are standard and are due, effectively, to Jacobi;

$$
\frac{2 K}{\pi} \mathrm{zs} u=\cot \frac{\pi u}{2 K}+4 \sum_{n=1}^{\infty} \frac{q^{2 n}}{1-q^{2 n}} \sin \frac{n \pi u}{K},
$$


and

$$
\frac{2 K}{\pi} \operatorname{ds} u=\frac{1}{\sin (\pi u / 2 K)}-4 \sum_{n=0}^{\infty} \frac{q^{2 n+1}}{1+q^{2 n+1}} \sin \left((n+1 / 2) \frac{\pi u}{K}\right),
$$

valid for $|\operatorname{Im} u|<\pi \operatorname{Re} K^{\prime} / 2$.

The notation is the usual elliptic one, with,

$$
q=e^{-\mu}=e^{-\pi K^{\prime} / K}, \quad q^{\prime}=e^{-\mu^{\prime}}=e^{-\pi K / K^{\prime}},
$$

and $K, K^{\prime}$ given by,

$$
K(k)=\int_{0}^{1} \frac{d z}{\sqrt{\left(1-z^{2}\right)\left(1-k^{2} z^{2}\right)}}, \quad K^{\prime}=K\left(k^{\prime}\right),
$$

where $k^{2}+k^{2}=1$.

In this scheme the key fact is that expansions of (29) and (30) in powers of $u$ will produce expressions for the quantities $\epsilon_{t}$ and $\eta_{t}$, of (8) and (20), in terms of $k$ and $K$. This is done by Glaisher who then proceeds to derive the relation to Eisenstein series, (9). Explicit forms are given later. For now we note the Laurent expansion of ds,

$$
\operatorname{ds} u=\frac{1}{u}+4 \sum_{l=1}^{\infty} \frac{(-1)^{l}}{(2 l-1) !}\left(\frac{\pi}{2 K}\right)^{2 l} \eta_{l} u^{2 l-1},
$$

exhibiting the partial energies, $\eta_{l}$, as coefficients.

Temperature inversion is just the interchange, $k \leftrightarrow k^{\prime}$ so that $K \leftrightarrow K^{\prime}$ and the effect can be deduced directly from (29) and (30) because the functions zs and ds reproduce themselves, i.e.

$$
\begin{aligned}
& \mathrm{zs}\left(u, k^{2}\right)=-i \mathrm{zs}\left(-i u, k^{2}\right)-\frac{\pi u}{2 K K^{\prime}} \\
& \mathrm{ds}\left(u, k^{2}\right)=-i \mathrm{ds}\left(-i u, k^{\prime 2}\right) .
\end{aligned}
$$

We might term this the Jacobian approach to inversion, and the one via Eisenstein series, the Weierstrassian approach.

Some inversion formulae are given in Whittaker and Watson [26] p.535 ex.60 derived from the imaginary transformation of sd. See also Hancock [27], p.308, Ex.4.

The expansions (29) and (30) yield, on setting $k \rightarrow k^{\prime}, u \rightarrow-i u$,

$$
\frac{2 K^{\prime}}{i \pi} \mathrm{zs}\left(-i u, k^{2}\right)=\operatorname{coth} \frac{\pi u}{2 K^{\prime}}-4 \sum_{n=1}^{\infty} \frac{q^{2 n}}{1-q^{\prime 2 n}} \sinh \frac{n \pi u}{K^{\prime}}
$$


and

$$
\frac{2 K^{\prime}}{i \pi} \mathrm{ds}\left(-i u, k^{2}\right)=\frac{1}{\sinh \left(\pi u / 2 K^{\prime}\right)}+4 \sum_{n=0}^{\infty} \frac{q^{2 n+1}}{1+q^{2 n+1}} \sinh \left((n+1 / 2) \frac{\pi u}{K^{\prime}}\right)
$$

so that from (33) and (34) we obtain the general inversion formula,

$$
\begin{aligned}
& K\left[\operatorname{coth} \frac{\pi u}{2 K^{\prime}}-4 \sum_{n=1}^{\infty} \frac{q^{\prime 2 n}}{1-q^{2 n}} \sinh \frac{n \pi u}{K^{\prime}}\right] \\
& =K^{\prime}\left[\cot \frac{\pi u}{2 K}+4 \sum_{n=1}^{\infty} \frac{q^{2 n}}{1-q^{2 n}} \sin \frac{n \pi u}{K}\right]+u,
\end{aligned}
$$

which reproduces (12) on expansion in $u$, after noting that $\mu=\pi K^{\prime} / K$ and $\mu^{\prime}=$ $\pi K / K^{\prime}$. We have said before that this is equivalent to the inversion symmetry (11).

As a special case, an elementary consequence of (11) is that $\epsilon_{t}(1)$ vanishes if $t$ is odd, $c f$ Glaisher [18], §§86-94. (This follows more directly from a consideration of the Eisenstein series.) It gives the interesting identity for the Bernoulli numbers,

$$
\sum_{n=1}^{\infty} \frac{n^{2 t-1}}{e^{2 \pi n}-1}=\frac{B_{2 t}}{4 t}, \quad t=3,5, \ldots
$$

rediscovered many times. Watson's proof, [28], involves an elliptic function expansion for $\mathrm{ds}^{2}$ similar to (29).

When $t=1$ the identity is modified to,

$$
\sum_{n=1}^{\infty} \frac{n}{e^{2 \pi n}-1}=\frac{B_{2}}{4}-\frac{1}{8 \pi}=\frac{1}{24}-\frac{1}{8 \pi}
$$

The 'extra' contribution of $1 / 8 \pi$ arises from the inhomogeneous part of the imaginary transformation of the $\zeta$-function, zs, (33) and corresponds, in our earlier terminology, to the zero mode on the circle, $\mathrm{S}^{1}$. For this special case the vacuum energy is,

$$
\bar{E}_{1}(\xi)=-\frac{B_{2}}{2}+\frac{\xi}{2 \pi}+2 \sum_{n=1}^{\infty} \frac{n}{e^{2 \pi n / \xi}-1} .
$$

The identity (38) can be derived via the full duality relation obtained from $(36)$,

$$
\mu^{\prime}\left(\frac{B_{2}}{2}-2 \sum_{n=1}^{\infty} \frac{n q^{2 n}}{1-q^{2 n}}\right)=-\mu\left(\frac{B_{2}}{2}-2 \sum_{n=1}^{\infty} \frac{n q^{2 n}}{1-q^{2 n}}\right)+\frac{1}{2 \pi}
$$


by setting $\mu=\mu^{\prime}$. Equations (36) and (40) seem to have been derived first by Schlömilch, [29]. Curiously he does not produce (12) although it is implicit in his formula.

Expressed in terms of the internal energy, (40) becomes,

$$
\bar{E}_{1}(\xi)-\frac{\xi}{2 \pi}=-\xi^{2} \bar{E}_{1}(1 / \xi)
$$

and we see that the zero modes have disappeared from the left hand side.

In terms of the Eisenstein series, (9), one encounters the series $G_{1}$ which formally diverges. Giving this term meaning, as described in Rademacher, for example, would produce the same result as above, but we have managed to bypass this analysis. Indeed, from (41), by recognising that the quantity possessing simple inversion properties is $\bar{E}_{1}(\xi)-\xi / 4 \pi$, one can derive the expression for $G_{1}$, [13] (63.82),

$$
G_{1}(1, i / \xi)=(2 \pi)^{2}\left(\frac{B_{2}}{2}-2 \sum_{n=1}^{\infty} \frac{n q^{2 n}}{1-q^{2 n}}+\frac{\xi}{4 \pi}\right) .
$$

\section{Spinors again.}

In this section we discuss the corresponding equations for fermions and also give results analogous to the recursive formulae (16) and (18) for bosons. To begin with, we present the fermion equivalents of the inversions (36) and (37).

The general inversion formula following from (30), (35) and (33),

$$
\begin{aligned}
& K\left[\frac{1}{\sinh \left(\pi u / 2 K^{\prime}\right)}+4 \sum_{n=0}^{\infty} \frac{q^{2 n+1}}{1+q^{2 n+1}} \sinh \left((n+1 / 2) \frac{\pi u}{K^{\prime}}\right)\right] \\
& \quad=K^{\prime}\left[\frac{1}{\sin (\pi u / 2 K)}-4 \sum_{n=0}^{\infty} \frac{q^{2 n+1}}{1+q^{2 n+1}} \sin \left((n+1 / 2) \frac{\pi u}{K}\right)\right]
\end{aligned}
$$

is equivalent to the Fermi-Dirac relation, (25) or (26). The identity analogous to (37) is,

$$
\sum_{n=0}^{\infty} \frac{(2 n+1)^{4 t+1}}{e^{(2 n+1) \pi}+1}=\frac{1}{4}\left(2^{4 t+1}-1\right) \frac{B_{4 t+2}}{2 t+1} .
$$

There is no zero mode special case. The absence is typical for spinor thermodynamical quantities in general.

In order to extract the $\eta_{t}$ from (32), the explicit Laurent expansion of $\mathrm{ds}$ is required. A number of ways of obtaining this are described in Appendix B. The one 
most appropriate at the moment depends on the differential equation satisfied by ds. The point is that the cubic recursion thence derived in Appendix B allows any $\eta_{t}$ to be expressed as polynomials in $\eta_{1}$ and $\eta_{2}$. This means that, in this case, the energies on the circle and three-sphere are sufficient to determine all energies. The reason for the contrast with scalars is the absence of an obstructing zero mode.

The equations corresponding to (16) are,

$$
\begin{aligned}
& \eta_{3}=\frac{240}{7} \eta_{1}\left(\eta_{2}+8 \eta_{1}^{2}\right) \\
& \eta_{4}=\frac{40}{3}\left(7 \eta_{2}^{2}+240 \eta_{1}^{2} \eta_{2}+576 \eta_{1}^{4}\right) \\
& \eta_{5}=\frac{3840}{11} \eta_{1}\left(85 \eta_{2}^{2}+960 \eta_{1}^{2} \eta_{2}+4032 \eta_{1}^{4}\right)
\end{aligned}
$$

and the spinor energies are, for example,

$$
\begin{aligned}
& \bar{E}_{1}^{\mathrm{f}}(\xi)=2 \eta_{1} \\
& \bar{E}_{3}^{\mathrm{f}}(\xi)=\frac{1}{2}\left(\eta_{2}-\eta_{1}\right) \\
& \bar{E}_{5}^{\mathrm{f}}(\xi)=\frac{1}{48}\left(9 \eta_{1}-10 \eta_{2}+\eta_{3}\right)
\end{aligned}
$$

allowing $\bar{E}_{5}^{\mathrm{f}}$ to be expressed in terms of $\bar{E}_{1}^{\mathrm{f}}(\xi)$ and $\bar{E}_{3}^{\mathrm{f}}(\xi)$, and this may be continued.

\section{Explicit formulae.}

The value $\xi=1$ is the lemniscate case and for scalars, e.g. [20] p.64,

$$
\epsilon_{2}(1)=\frac{\Gamma^{8}(1 / 4)}{5120 \pi^{6}}, \quad \epsilon_{3}(1)=0
$$

In elliptic function terminology, the lemniscate value corresponds to $K=K^{\prime}$ and so to a modulus of $1 / \sqrt{2}$.

This is a specially interesting point, but it is an important fact in the practical application of elliptic functions that, given the value of $K / K^{\prime}$, one can calculate the values of $K$ and $K^{\prime}$ separately, and also of $k$. The physical significance of this for us is the following.

Expansion of the right-hand sides of (29) and (30) in powers of $u$ introduces the quantities $\epsilon_{t}$ and $\eta_{t}$, as has already been used to derive inversion symmetry. However now we see that the left-hand sides will yield expressions for $\epsilon_{t}$ and $\eta_{t}$ in 
terms of $k$ and $K$. These have been given by Glaisher, for example. Hence, knowing the temperature, i.e. $\xi=K / K^{\prime}$, one can compute the partial energies from elliptic function numerics. Furthermore, according to our previous analysis, see (18) and (44), only $\epsilon_{2}, \epsilon_{3}, \eta_{1}$ and $\eta_{2}$ need to be evaluated in this way in order that all spheres are encompassed, in principle.

Making use of Glaisher's expressions, for convenience, we find, for bosons,

$$
\begin{aligned}
& \epsilon_{2}=\frac{1}{15 \pi^{4}}\left(1-k^{2}+k^{4}\right) K^{4} \\
& \epsilon_{3}=\frac{4}{63 \pi^{6}}\left(2-k^{2}\right)\left(2 k^{2}-1\right)\left(1+k^{2}\right) K^{6} .
\end{aligned}
$$

As might be expected, the expansions occur elsewhere. The relation between the Eisenstein series and the elliptic functions follows from the very basic connection between Weierstrass and Jacobi, e.g. [20] p.24,

$$
\wp u=\frac{4 K^{2}}{\operatorname{sn}^{2}(2 K u)}-\frac{4}{3}\left(1+k^{2}\right) K^{2},
$$

and power series expansion of the right-hand side again yields the $(k, K)$ polynomials in (45). The expansion of $\wp u$ is given by (13) as a power series in $g_{2}$ and $g_{3}$, the standard expressions for which are, [20], pp.59-60, (with a sign error), [19] IV p.92, [30] p.152, [27] pp.201, 298, [31], p.57, [32] p.384,

$$
\begin{aligned}
& g_{2}=\frac{4}{3} \lambda^{2}\left(1-k^{2}+k^{4}\right)=\frac{4}{3} \lambda^{2}\left(1-\left(k k^{\prime}\right)^{2}\right) \\
& g_{3}=\frac{8}{27} \lambda^{3}\left(1+k^{2}\right)\left(1-2 k^{2}\right)\left(1-k^{2} / 2\right)=\frac{8}{27} \lambda^{3}\left(k^{\prime 2}-k^{2}\right)\left(1+\frac{1}{2}\left(k k^{\prime}\right)^{2}\right),
\end{aligned}
$$

where $\lambda$ is defined by

$$
\lambda=\left(\frac{2 K}{\omega_{1}}\right)^{2} .
$$

Note that our periods are $\omega_{1}$ and $\omega_{2}$ and we are setting $\omega_{1}=1$ and $\omega_{2}=i K^{\prime} / K$. For useful relational facts see [19] vol.II, chap.IV, pp.180,207, and [20],pp.44-46.

It is interesting to confirm the inversion symmetry directly from the forms in (45). Write generally $\epsilon_{t}(k, K)=F_{t}\left(k^{2}\right) K^{2 t}(k)$. Inversion is $k^{2} \rightarrow k^{\prime 2}=1-k^{2}$. Then (Hancock [27] p.308, Ex.4),

$$
F_{t}\left(1-k^{2}\right)=(-1)^{t} F_{t}\left(k^{2}\right)
$$

so that,

$$
\epsilon_{t}\left(k^{\prime}, K^{\prime}\right)=(-1)^{t}\left(\frac{K}{K^{\prime}}\right)^{2 t} \epsilon_{t}(k, K)
$$


which is exactly (11).

Turning now to the fermion case, the expressions we need are most easily read off from the expansion for ds given in Glaisher, [18],

$$
\begin{aligned}
& \eta_{1}=-\frac{\lambda}{24 \pi^{2}}\left(1-2 k^{2}\right) \\
& \eta_{2}=\frac{\lambda^{2}}{240 \pi^{4}}\left(7+8 k^{2}-8 k^{4}\right) \\
& \eta_{3}=-\frac{\lambda^{3}}{504 \pi^{6}}\left(1-2 k^{2}\right)\left(16 k^{4}-16 k^{2}+31\right),
\end{aligned}
$$

showing, as a simple check, that $\eta_{1}(1)=0, \eta_{3}(1)=0$ at the lemniscate point, $k=1 / \sqrt{2}$. The expressions satisfy the relation in (43).

The power series expansions of the elliptic functions used here, although old and standard, are not emphasised in the usual textbooks, with a few exceptions. For this reason they are developed a little in Appendix B, where a recursion for the expansion coefficients of $\mathrm{ds}$ is given and has been checked against Glaisher's expressions.

\section{Numerical evaluation. Singular moduli.}

Computationally, the nome, $q$, is known from the temperature so the modulus, $k$, can be calculated, usually via a power series in $q$, and then $K(k)$ follows straightforwardly by the Legendre-Landen-Gauss method (e.g. Appell and Lacour, [32] §198, Hurwitz and Courant, [21] p.242, Greenhill [31], p.322) or one could, pragmatically, just use the built-in algorithms of Mathematica.

Generally, there seems no advantage in using an elliptic formulation, as opposed to a simple evaluation of the defining thermal state summations, (8) and (20), taken in conjunction with inversion, (11) and (25). However, as well as the lemniscate case, there are many other special values of $K^{\prime} / K$ that have 'exact' expressions for $k$ so that only the computation of $K$ from $k$ remains and in many cases, like the lemniscate one, $K$ is known in closed form.

Abel has shown, for $K^{\prime} / K$ of the special form,

$$
\frac{K^{\prime}}{K}=\frac{a+b \sqrt{n}}{c+d \sqrt{n}}, \quad a, b, c, d, n \in \mathbb{Z},
$$

that $k$ is the solution of an algebraic equation with integer coefficients. Relatedly, it is known that this also applies when $K^{\prime} / K=\sqrt{N}$, where $N$ is rational. In 
particular, when $N$ is an integer, the modulus, $k \equiv k_{N}$, is referred to as a singular modulus.

There are many classic works on modular equations and singular moduli. A little known one that gives a direct algebraic construction for prime order is Russell, [33]. Lists of $k_{N}$ can be found, e.g. in Weber [30,34] and in Greenhill [35]. The notebooks of Ramanujan also contain many examples of modular equations, [36].

It is also known that the complete elliptic integral, $K$, evaluated at a singular modulus can be expressed in terms of Gamma functions with rational arguments. The $N=1,3,4$ cases are classic, (e.g. [26]) and the $N=2$ case is treated by Glasser and Wood [37], although it seems to be implicit in work of Ramanujan.

The general case was discussed by Selberg and Chowla, [38], who proved that the complete elliptic integral, $K\left(k_{N}\right)$, is always expressible in terms of Gamma functions and gave the $N=5$ and $N=7$ forms.

The lowest value, $N=1$, is just the lemniscate value touched upon earlier. When $N$ is a square, $K\left(k_{N}\right)$ is an algebraic number times $K\left(k_{1}\right)$. More generally when $N$ contains a square, $K\left(k_{N}\right)$ is an algebraic number times $K\left(k_{N_{0}}\right)$ where $N_{0}$ is the square-free part of $N$.

Much effort has gone into finding $K\left(k_{N}\right)$ together with $k_{N}$ and, by now, many specific examples have been listed e.g. [37-45]. Borwein and Zucker, [44], show that using the beta function often provides simplified expressions.

In the following we calculate closed forms of the energy in one, three and five dimensions for a selection of values, $\xi_{N}$, of $\xi$ that correspond to singular moduli.

Examples we will look at are $N=1,2,3,6,7,10,15$, all of which can be found in Zucker and Joyce, [43], for example. For convenience we summarize the singular moduli used,

$$
\begin{aligned}
k_{1} & =\frac{1}{\sqrt{2}} \\
k_{2} & =\sqrt{2}-1 \\
k_{3} & =\frac{1}{4} \sqrt{2}(\sqrt{3}-1) \\
k_{6} & =(\sqrt{3}-\sqrt{2})(2-\sqrt{3}) \\
k_{7} & =\frac{1}{8} \sqrt{2}(3-\sqrt{7}) \\
k_{10} & =(\sqrt{10}-3)(\sqrt{2}-1)^{2} \\
k_{15} & =\frac{1}{8 \sqrt{2}}(2-\sqrt{3})(\sqrt{5}-\sqrt{3})(3-\sqrt{5}),
\end{aligned}
$$


and for $K\left(k_{N}\right)$, correspondingly,

$$
\begin{aligned}
K\left(k_{1}\right) & =\frac{1}{4} \beta(1 / 4) \\
K\left(k_{2}\right) & =\frac{2^{3 / 4}}{16} \beta(1 / 8) \\
K\left(k_{3}\right) & =\frac{2^{1 / 3} 3^{1 / 4}}{12} \beta(1 / 6) \\
K\left(k_{6}\right) & =\frac{2^{1 / 12} 3^{1 / 4}}{48}(\sqrt{2}-1)(\sqrt{3}+1) \beta(1 / 24) \\
K\left(k_{7}\right) & =\frac{2^{5 / 7} 7^{3 / 4}}{14} \frac{\beta(1 / 7) \beta(2 / 7)}{\beta(1 / 14)} \\
K\left(k_{10}\right) & =\frac{10^{1 / 4}}{80}(\sqrt{5}-2)^{1 / 2} \frac{\beta(1 / 40) \beta(9 / 40)}{\beta(3 / 8)} \\
K\left(k_{15}\right) & =\frac{15^{1 / 4}}{60}(\sqrt{5}-1) \frac{\beta(1 / 15) \beta(4 / 15)}{\beta(1 / 3)},
\end{aligned}
$$

where the beta function,

$$
\beta(x) \equiv B(x, x)=\frac{\Gamma^{2}(x)}{\Gamma(2 x)},
$$

has been used.

The energies can be calculated at the values $\xi=\xi_{N}=1 / \sqrt{N}$. We give below the first three values, the simpler remaining ones being relegated to Appendix C. The scalar closed expressions in three dimensions are, from (18) and (45),

$$
\begin{aligned}
& \bar{E}_{3}\left(\xi_{1}\right)=\frac{\Gamma^{8}(1 / 4)}{5120 \pi^{6}} \\
& \bar{E}_{3}\left(\xi_{2}\right)=\frac{(3-2 \sqrt{2})}{3072 \pi^{2}} \frac{\Gamma^{4}(1 / 8)}{\Gamma^{4}(5 / 8)} \\
& \bar{E}_{3}\left(\xi_{3}\right)=\frac{\Gamma^{4}(1 / 6) \Gamma^{4}(1 / 3)}{12288 \pi^{6}} .
\end{aligned}
$$

and in five dimensions,

$$
\begin{aligned}
& \bar{E}_{5}\left(\xi_{1}\right)=-\frac{\Gamma^{8}\left(\frac{1}{4}\right)}{61440 \pi^{6}} \\
& \bar{E}_{5}\left(\xi_{2}\right)=\frac{\Gamma^{8}\left(\frac{1}{8}\right)\left((7-5 \sqrt{2}) \Gamma^{4}\left(\frac{1}{8}\right)+24(-3+2 \sqrt{2}) \pi^{2} \Gamma^{2}\left(\frac{1}{4}\right)\right)}{7077888 \pi^{6} \Gamma^{6}\left(\frac{1}{4}\right)} \\
& \bar{E}_{5}\left(\xi_{3}\right)=-\frac{\left(\Gamma^{6}\left(\frac{1}{6}\right)\left(562^{\frac{2}{3}} \pi^{4}+11 \Gamma^{6}\left(\frac{1}{3}\right)\right)\right)}{24772608 \pi^{9}} .
\end{aligned}
$$


The spinor energies are given by (44). In one dimension,

$$
\begin{aligned}
& \bar{E}_{1}^{\mathrm{f}}\left(\xi_{1}\right)=0 \\
& \bar{E}_{1}^{\mathrm{f}}\left(\xi_{2}\right)=\frac{(5-4 \sqrt{2}) \Gamma^{2}\left(\frac{1}{8}\right)}{96 \pi \Gamma^{2}\left(\frac{5}{8}\right)} \\
& \bar{E}_{1}^{\mathrm{f}}\left(\xi_{3}\right)=-\frac{\left(\Gamma^{2}\left(\frac{1}{6}\right) \Gamma^{2}\left(\frac{1}{3}\right)\right)}{96 \pi^{3}},
\end{aligned}
$$

and in three,

$$
\begin{aligned}
& \bar{E}_{3}^{\mathrm{f}}\left(\xi_{1}\right)=\frac{3 \Gamma^{8}\left(\frac{1}{4}\right)}{2560 \pi^{6}} \\
& \bar{E}_{3}^{\mathrm{f}}\left(\xi_{2}\right)=\frac{\Gamma^{4}\left(\frac{1}{8}\right)\left((-21+16 \sqrt{2}) \Gamma^{4}\left(\frac{1}{8}\right)+32(8-5 \sqrt{2}) \pi^{2} \Gamma^{2}\left(\frac{1}{4}\right)\right)}{49152 \pi^{4} \Gamma^{4}\left(\frac{1}{4}\right)} \\
& \bar{E}_{3}^{\mathrm{f}}\left(\xi_{3}\right)=\frac{1}{3072 \pi^{6}}\left(\Gamma^{4}\left(\frac{1}{6}\right) \Gamma^{4}\left(\frac{1}{3}\right)+\frac{384 \pi^{5} \Gamma^{2}\left(\frac{7}{6}\right)}{\Gamma^{2}\left(\frac{2}{3}\right)}\right) .
\end{aligned}
$$

In five dimensions, simpler spinor examples are,

$$
\begin{aligned}
& \bar{E}_{5}^{\mathrm{f}}\left(\xi_{1}\right)=-\frac{32 \Gamma^{8}\left(\frac{5}{4}\right)}{\pi^{6}} \\
& \bar{E}_{5}^{\mathrm{f}}\left(\xi_{3}\right)=-\frac{\left(3362^{\frac{1}{3}} \pi^{8}+702^{\frac{2}{3}} \pi^{4} \Gamma^{6}\left(\frac{1}{3}\right)+5 \Gamma^{12}\left(\frac{1}{3}\right)\right)}{96768 \pi^{6} \Gamma^{6}\left(\frac{2}{3}\right)} .
\end{aligned}
$$

We now present some numbers computed from the above formulae which, incidentally, agree well with a direct evaluation using the elliptic numerical functions available in Mathematica. As a check, for increasing $N$, i.e. decreasing temperature, the values approach the standard Casimir values, which are usually expressed in Bernoulli numbers. This could be thought of only as a necessary confirmation that the second terms in (8) and (20) must tend to zero as $\xi$ becomes smaller. However, it does mean that however complicated the closed forms become for large $N$, they must ultimately tend to a Bernoulli expression i.e. a simple rational number! The zero temperature quantities are completely entangled in the finite form expression. 
For scalars,

$$
\begin{array}{rlll}
\bar{E}_{3}\left(\xi_{1}\right) & =0.006065678717786288844 & \bar{E}_{5}\left(\xi_{1}\right) & =-0.0005054732264821907370 \\
\bar{E}_{3}\left(\xi_{2}\right) & =0.004305183178876425232 & \bar{E}_{5}\left(\xi_{2}\right) & =-0.0005125278116483441533 \\
\bar{E}_{3}\left(\xi_{3}\right) & =0.004185448373868254025 & \bar{E}_{5}\left(\xi_{3}\right) & =-0.0005125654321803054872 \\
\bar{E}_{3}\left(\xi_{6}\right) & =0.004166873656512456887 & \bar{E}_{5}\left(\xi_{6}\right) & =-0.0005125661374804481332 \\
\bar{E}_{3}\left(\xi_{7}\right) & =0.004166726978035004716 & \bar{E}_{5}\left(\xi_{7}\right) & =-0.0005125661375588626478 \\
\bar{E}_{3}\left(\xi_{10}\right) & =0.004166669015900687631 & \bar{E}_{5}\left(\xi_{10}\right) & =-0.0005125661375661265283 \\
\bar{E}_{3}\left(\xi_{15}\right) & =0.004166666693680499456 & \bar{E}_{5}\left(\xi_{15}\right) & =-0.0005125661375661375647 \\
\bar{E}_{3}\left(\xi_{\infty}\right) & =0.0041 \dot{6} & \bar{E}_{5}\left(\xi_{\infty}\right) & =-0.00051256613756 \dot{6} \\
& =1 / 240 & & =-31 / 6480 .
\end{array}
$$

For spinors,

$$
\begin{aligned}
& \bar{E}_{3}^{\mathrm{f}}\left(\xi_{1}\right)=0.03639407230671773306 \quad \bar{E}_{1}^{\mathrm{f}}\left(\xi_{1}\right)=0 \\
& \bar{E}_{3}^{\mathrm{f}}\left(\xi_{2}\right)=0.03543620656643122772 \quad \bar{E}_{1}^{\mathrm{f}}\left(\xi_{2}\right)=-0.06007307862716140024 \\
& \bar{E}_{3}^{\mathrm{f}}\left(\xi_{3}\right)=0.03541764326165557577 \quad \bar{E}_{1}^{\mathrm{f}}\left(\xi_{3}\right)=-0.07470339906473023869 \\
& \bar{E}_{3}^{\mathrm{f}}\left(\xi_{6}\right)=0.03541666779673327755 \quad \bar{E}_{1}^{\mathrm{f}}\left(\xi_{6}\right)=-0.08242382467179113065 \\
& \bar{E}_{3}^{\mathrm{f}}\left(\xi_{7}\right)=0.03541666684440446497 \quad \bar{E}_{1}^{\mathrm{f}}\left(\xi_{7}\right)=-0.08242382467179113065 \\
& \bar{E}_{3}^{\mathrm{f}}\left(\xi_{10}\right)=0.03541666666803304403 \quad \bar{E}_{1}^{\mathrm{f}}\left(\xi_{10}\right)=-0.08242382467179113065 \\
& \bar{E}_{3}^{\mathrm{f}}\left(\xi_{15}\right)=0.03541666666666835152 \quad \bar{E}_{1}^{\mathrm{f}}\left(\xi_{15}\right)=-0.08332293842073511712 \\
& \bar{E}_{3}^{\mathrm{f}}\left(\xi_{\infty}\right)=0.03541 \dot{6} \quad \bar{E}_{1}^{\mathrm{f}}\left(\xi_{\infty}\right)=-0.08 \dot{3} \\
& =17 / 48 \quad=-1 / 12 \text {. }
\end{aligned}
$$

These numbers also show that the range, $0 \leq \xi \leq 1$, is essentially a Casimir range. Inversion can be used to obtain values in the complementary 'high temperature' region, $1 \leq \xi \leq \infty$.

\section{Conclusion and extensions.}

We have presented expressions for the internal energies of conformally coupled scalars and spinors on odd spheres in terms of elliptic function quantities. They can be used for convenient numerical evaluation, but our main aim, which has become somewhat overshadowed, was really to investigate the question of temperature inversion symmetry. Regarding this, it was only for the three-sphere that an overall symmetric quantity (the total energy) could be found. For the others only the "partial energies' satisfied the symmetry. The same structure is noticed by Kutasov and 
Larsen, [46]. Thus the statements in Cardy, [3], while correct, are, possibly, slightly misleading. See also Oshima [47].

The closed form expressions at temperatures corresponding to singular moduli are perhaps somewhat academic. They correspond to denominators in the thermal $\zeta$-function, [1], of the form $n^{2}+N m^{2}$ indicating number theory involvement, [48], and hence elliptic functions. Everything could be obtained from the Epstein form of the thermal $\zeta$-function. (See related calculations by Kennedy [49] on $S^{3} / \Gamma$.) Indeed it was by the use of the known 'exact' forms of certain Epstein functions that Zucker derived values of $K\left(k_{N}\right)$. Most other calculations also use Kronecker's limit formula, following Selberg and Chowla, [38]. It thus seems that our discussion is a little circular but it is systematic and has the advantage of uniformity of treatment. One must also not forget that there seem to be purely algebraic ways of obtaining $K\left(k_{N}\right)$, [36], independent of the limit formula. Not only that but it would be otherwise difficult to treat $K^{\prime} / K$ of the more general Abel structure (50).

As further justification of our elliptic approach, we draw attention to the fact that the internal energy on the arbitrary odd sphere, $\mathrm{S}^{d}$, is a polynomial in just two quantities, see (18) and (44).

For scalars this is a simple consequence of standard analytical properties of the Weierstrass function, $\wp$, but seems not so obvious in the Epstein formulation.

For spinors, the explicit construction of a cubic recursion seems to be new. It is most easily derived directly from the differential equation satisfied by ds.

There are a large number of relations that we have not brought into the present discussion. For example, the double sum representations of thermodynamic quantities obtained in the earlier work [50] and [25] can be manipulated. One of the summations can be performed yielding a sum of inverse powers of hyperbolic functions. These constitute adequate numerical expressions and also can be turned into elliptic function form to make contact with the present approach, $c f$ [51].

Regarding even spheres, no direct relation to elliptic functions could be detected. The sums relevant for standard thermodynamic scalars (bosons) are

$$
\sum_{n=0}^{\infty}(2 n+1)^{2 t} \frac{q^{2 n+1}}{1-q^{2 n+1}} .
$$

An obvious generating function, in the spirit of odd spheres, is

$$
\sum_{n=0}^{\infty} \frac{q^{2 n+1}}{1-q^{2 n+1}} \cos \left((n+1 / 2) \frac{\pi u}{K}\right)
$$


with periodicity $4 K$, but no periodicity in the $i K^{\prime}$ direction. As it stands, this makes a connection with Jacobian elliptic functions impossible and again indicates the necessity of altering the thermal periodicity. The same statements hold for spinors. In future work we intend to investigate this question.

We will also investigate a general elliptic description of the complete thermodynamics of the system, not just the internal energy.

Finally, regarding references, we have not been able to consult some classic texts, such as those by Enneper, Durège and Jordan on elliptic functions.

Acknowledgment: KK was supported by the Max-Planck-Institute for Mathematics in the Sciences, Leipzig. 


\section{Appendix A. Fermion expressions.}

As might be expected in a topic involving elliptic functions there are many routes to required results. This will be illustrated in this appendix by relating fermion and boson quantities. While not absolutely necessary, the analysis usefully invokes several basic pieces of elliptic technology. There are various ways of writing and re-arranging the fermion quantities.

In terms of the Weierstrass approach via the twisted $\widetilde{\wp},(27)$, one can seek to bring back the ordinary Weierstrass function, $\wp$, by re-arranging the summation lattice in (27). If this is drawn out, it is seen that the summations divide into a positive and a negative square lattice, displaced, one from the other, by, say, $\omega_{1}$ along the real axis and both rotated by $45^{\circ}$ with respect to the original $m_{1}, m_{2}$ lattice. Therefore define new summation integers by $M=m_{1}+m_{2}$ and $m=m_{1}-m_{2}$. The positive lattice corresponds to $M$ and $m$ both being even, and the negative lattice to $M$ and $m$ both odd. Simple algebra then gives,

$$
\widetilde{\wp}\left(u ; \omega_{1}, \omega_{2}\right)=\wp\left(u ; \widetilde{\omega}_{1}, \widetilde{\omega}_{2}\right)-\wp\left(u+\omega_{1} ; \widetilde{\omega}_{1}, \widetilde{\omega}_{2}\right)+\wp\left(\omega_{1} ; \widetilde{\omega}_{1}, \widetilde{\omega}_{2}\right),
$$

where

$$
\begin{aligned}
& \widetilde{\omega}_{1}=\omega_{1}-\omega_{2} \\
& \widetilde{\omega}_{2}=\omega_{1}+\omega_{2}
\end{aligned}
$$

so that $\omega_{1}=\left(\widetilde{\omega}_{2}+\widetilde{\omega}_{1}\right) / 2$ is a half-period of $\wp\left(u ; \widetilde{\omega}_{1}, \widetilde{\omega}_{2}\right)$. This function has a negative discriminant. The scaled rotation, (52), has determinant 2.

The same transformation can be applied to the normal $\wp$-function, to give

$$
\wp\left(u ; \omega_{1}, \omega_{2}\right)=\wp\left(u ; \widetilde{\omega}_{1}, \widetilde{\omega}_{2}\right)+\wp\left(u+\omega_{1} ; \widetilde{\omega}_{1}, \widetilde{\omega}_{2}\right)-\wp\left(\omega_{1} ; \widetilde{\omega}_{1}, \widetilde{\omega}_{2}\right) .
$$

Combined with (51) this yields,

$$
\widetilde{\wp}\left(u ; \omega_{1}, \omega_{2}\right)=2 \wp\left(u ; \widetilde{\omega}_{1}, \widetilde{\omega}_{2}\right)-\wp\left(u ; \omega_{1}, \omega_{2}\right),
$$

which can be used to give an expansion of the fermion $\widetilde{\wp} u$ in terms of that for $\wp u$. Thus, similarly to (13), we write,

$$
\wp\left(u ; \widetilde{\omega}_{1}, \widetilde{\omega}_{2}\right)=\frac{1}{u^{2}}+\sum_{t=2}^{\infty}(2 t-1) \widetilde{G}_{t} u^{2 t-2},
$$

so that,

$$
\widetilde{\wp}\left(u ; \omega_{1}, \omega_{2}\right)=\frac{1}{u^{2}}+\sum_{t=2}^{\infty}(2 t-1)\left(2 \widetilde{G}_{t}-G_{t}\right) u^{2 t-2},
$$


and therefore

$$
H_{t}=2 \widetilde{G}_{t}-G_{t},
$$

allowing $\eta_{t}$ to be found from (23). This will be taken further in Appendix B.

Relation (54) expresses the fermion function, $\widetilde{\wp}$, in terms of the boson one, $\wp$, which is sometimes useful.

Introducing yet another, more flexible notation, equivalent to the zs function is the theta function construction ( $c f$ [19], vol.III p.117),

$$
2 K \mathrm{zs}(2 K u) \equiv \Phi(u, \tau)=\frac{d}{d u} \log \theta_{1}(u, \tau),
$$

with

$$
q=e^{i \pi \tau}=e^{-\mu}=e^{-\pi K^{\prime} / K} .
$$

Thomae denotes $\Phi$ by $Z_{11}$, [27] p.295.

In terms of $\Phi$, the fermion function, ds, can be written formally as the difference of two composite expressions, ( $c f[13], \S \S 84,85)$,

$$
\begin{aligned}
2 K \operatorname{ds}(2 K u) & =\frac{1}{2} \Phi(u / 2,(1+\tau) / 2)-\frac{1}{2} \Phi((u+1) / 2,(1+\tau) / 2) \\
& =\frac{d}{d u} \log \frac{\theta_{1}(u / 2,(1+\tau) / 2)}{\theta_{2}(u / 2,(1+\tau) / 2)} \\
& =\frac{d}{d u} \log \frac{\theta_{1}(u / 2, \tau) \theta_{3}(u / 2, \tau)}{\theta_{2}(u / 2, \tau) \theta_{0}(u / 2, \tau)} .
\end{aligned}
$$

The last equality is a consequence of the quadratic Gauss transformation expressed in theta functions, (e.g. [19] II, pp.119,120,207). This can be confirmed by combining the relevant $q$-series by eye (e.g. [19] III,p.117, [20], p.431, [18]) and this leads onto another way of relating fermion and boson expressions which is to start from the left-hand side of the relation (22), and work at the $q$-series level by applying, $\grave{a}$ la Jacobi, the trivial, and common, identity,

$$
\frac{x}{1+x}=\frac{x}{1-x}-\frac{2 x^{2}}{1-x^{2}}
$$

to the quantities $\eta_{t}(\xi)$ of (20), which we can write as

$$
\eta_{t}(\xi)=2^{2 t-1} \frac{B_{2 t}(1 / 2)}{4 t}+\sum_{n=0}^{\infty} \frac{(2 n+1)^{2 t-1} q^{2 n+1}}{1+q^{2 n+1}} .
$$


The sum over odds is written as,

$$
\sum_{n=0}^{\infty} \frac{(2 n+1)^{2 t-1} q^{2 n+1}}{1+q^{2 n+1}}=\sum_{n=1}^{\infty} \frac{n^{2 t-1} q^{n}}{1+q^{n}}-\sum_{n=1}^{\infty} \frac{(2 n)^{2 t-1} q^{2 n}}{1+q^{2 n}}
$$

and (60) used on both parts, to give for the right-hand side,

$$
\sum_{n=1}^{\infty} \frac{n^{2 t-1} q^{n}}{1-q^{n}}-\sum_{n=1}^{\infty} \frac{(2 n)^{2 t-1} q^{2 n}}{1-q^{2 n}}-2 \sum_{n=1}^{\infty} \frac{n^{2 t-1} q^{2 n}}{1-q^{2 n}}+2 \sum_{n=1}^{\infty} \frac{(2 n)^{2 t-1} q^{4 n}}{1-q^{4 n}} .
$$

Then, simple algebra, yields the relation,

$$
\begin{aligned}
\eta_{t}(\xi) & =\epsilon_{t}(2 \xi)-2 \epsilon_{t}(\xi)-2^{2 t-1}\left(\epsilon_{t}(\xi)-2 \epsilon_{t}(\xi / 2)\right) \\
& =\Omega_{t}(2 \xi)-2 \Omega_{t}(\xi) \\
& =\Xi_{t}(\xi)-2\left(1+2^{2 t-2}\right) \epsilon_{t}(\xi)
\end{aligned}
$$

where $\Omega_{t}(\xi)=\epsilon_{t}(\xi)-2^{2 t-1} \epsilon_{t}(\xi / 2)$ and $\Xi(\xi)=\epsilon_{t}(2 \xi)+2^{2 t} \epsilon_{t}(\xi / 2)$. The combination (61) possesses the correct inversion behaviour, as a check. The two parts on the last line possess the inversion property separately. In terms of $q$, the right-hand side involves $q^{2}, q^{1 / 2}$ and $q$.

This expression can also be obtained by reorganising the summations in the Eisenstein series, $H_{2 k}$, and using the basic equation, (23).

In the lowest case of the circle, $t=1$, it is easily confirmed that the zero mode element of the scalar energy typically cancels on the right-hand side to leave exactly the same combination for the total fermion energy, $\bar{E}_{1}^{\mathrm{f}}$,

$$
\bar{E}_{1}^{\mathrm{f}}(\xi)=\bar{E}_{1}(2 \xi)-4 \bar{E}_{1}(\xi)+4 \bar{E}_{1}(\xi / 2) .
$$

Some relevant calculations on the relation between $\widetilde{\wp}$ and $\wp$ can be found in Tannery and Molk, [19] IV, pp.37-40. In our notation they have,

$$
\wp\left(u ; \omega_{1}, \omega_{2}\right)=\wp\left(u ; \widetilde{\omega}_{1}, \widetilde{\omega}_{2}\right)-\frac{\left(\widetilde{e}_{2}-\widetilde{e}_{1}\right)\left(\widetilde{e}_{2}-\widetilde{e}_{3}\right)}{\wp\left(u ; \widetilde{\omega}_{1}, \widetilde{\omega}_{2}\right)-\widetilde{e}_{2}},
$$

which also follows from (51), and leads to another form for $\widetilde{\wp}$,

$$
\widetilde{\wp}\left(u ; \omega_{1}, \omega_{2}\right)=\wp\left(u ; \widetilde{\omega}_{1}, \widetilde{\omega}_{2}\right)+\frac{\left(\widetilde{e}_{2}-\widetilde{e}_{1}\right)\left(\widetilde{e}_{2}-\widetilde{e}_{3}\right)}{\wp\left(u ; \widetilde{\omega}_{1}, \widetilde{\omega}_{2}\right)-\widetilde{e}_{2}} .
$$

We have not used this although it does yield the required expansions after, say, the introduction of two Jacobi functions, e.g. $\widetilde{\mathrm{sn}}^{2}$ and $\widetilde{\mathrm{dc}}^{2}$. 
It is possible to avoid the new modulus, $\widetilde{k}$, by repeating the transformation to the periods, $\widetilde{\omega}_{1}, \widetilde{\omega}_{2}$. This effectively amounts to doubling the periods to $2 \omega_{2}, 2 \omega_{1}$, which are periods of $\widetilde{\wp}$. The necessary analysis is given by Tannery and Molk [19] IV pp.276-280. Their quantities $y, Y$ and $Z$ are, in our notation,

$$
Y=\wp\left(u ; \omega_{1}, \omega_{2}\right), \quad y=\wp\left(u ; \widetilde{\omega}_{1}, \widetilde{\omega}_{2}\right), \quad Z=\wp\left(u ; 2 \omega_{1}, 2 \omega_{2}\right)
$$

so that the fermion $\widetilde{\wp}$ is,

$$
\widetilde{\wp}\left(u ; \omega_{1}, \omega_{2}\right)=2 y-Y
$$

Now $y$ can be expressed rationally in terms of $Z$ which leads to,

$$
\widetilde{\wp}=2 Z+2 \frac{\left(\mathrm{e}_{2}-\mathrm{e}_{1}\right)\left(\mathrm{e}_{2}-\mathrm{e}_{3}\right)}{Z-\mathrm{e}_{2}}-Y
$$

where the $\mathrm{e}_{i}$ are the roots associated with $Z$. By homogeneity $\mathrm{e}_{i}=e_{i} / 4$ and clearly the modulus associated with $Z$ remains $k$. Equation (65) can provide another way to expand the $\widetilde{\wp}$.

As a side remark, since $\widetilde{\wp}(u)$ has periods $2 \omega_{1}, 2 \omega_{2}$, it can be expressed rationally in terms of $Z$. We find, using the expression for $\wp(2 u)$ in terms of $\wp(u)$,

$$
\widetilde{\wp}\left(u ; \omega_{1}, \omega_{2}\right)=-\frac{4 Z^{4}+4 \gamma_{2} Z^{2}+10 \gamma_{3} Z+\gamma_{2}^{2} / 4}{4 Z^{3}-\gamma_{2} Z-\gamma_{3}}+2 \frac{\left(\mathrm{e}_{2}-\mathrm{e}_{1}\right)\left(\mathrm{e}_{2}-\mathrm{e}_{3}\right)}{Z-\mathrm{e}_{2}}
$$

where $\gamma_{2}$ and $\gamma_{3}$ are the invariants associated with $Z$. Homogeneity allows everything to be written in terms of $\wp_{\circ} \equiv \wp\left(u / 2 ; \omega_{1}, \omega_{2}\right)$. Thus

$$
Z(u)=\frac{1}{4} \wp_{\circ}, \quad \gamma_{2}=\frac{1}{16} g_{2}, \quad \gamma_{3}=\frac{1}{64} g_{3},
$$

and so

$$
\widetilde{\wp}\left(u ; \omega_{1}, \omega_{2}\right)=-\frac{1}{4} \frac{4 \wp_{\circ}^{4}+4 g_{2} \wp_{\circ}^{2}+10 g_{3} \wp_{\circ}+g_{2}^{2} / 4}{4 \wp_{\circ}^{3}-g_{2} \wp_{\circ}-g_{3}}+\frac{\left(e_{2}-e_{1}\right)\left(e_{2}-e_{3}\right)}{2\left(\wp_{\circ}-e_{2}\right)} .
$$

This does not seem to have any computational advantages. 


\section{Appendix B. The power series expansion of elliptic functions.}

For our purposes in this paper, we need the expansions of zs and ds. Glaisher, for example, lists all the expansions but it might be useful to present an outline of some derivations and other pertinant information.

The expansion of the elliptic functions sn $u$ etc. as power series in $u$ formed the subject of Weierstrass' first major work dating to 1840. The derivation is a technical issue that is not treated in much detail in most textbooks. Exceptions include Fricke, [52] and Briot and Bouquet [45].

There are many equivalent approaches depending on the representations of the elliptic functions employed. For example, one could use the theta form of zs, given later, or one could employ the relation between zs and the Weierstrass $\zeta$-function,

$$
\text { zs } u=\zeta u-\frac{\eta u}{K}, \quad \operatorname{zs}^{\prime} u=-\wp u+\frac{\eta}{K}
$$

The calculations for sn, cn and dn are described in Fricke, [52] pp.396+, and Königsberger, [53], following Weierstrass. There are two basic approaches. The first is a direct application of Taylor series. By successive differentiation, Jacobi showed that, for example,

$$
\frac{d^{2 \nu} \operatorname{sn} u}{d u^{2 \nu}}=\left(a_{0}^{(\nu)}+a_{1}^{(\nu)} \operatorname{sn}^{2} u+\ldots+a_{\nu}^{(\nu)} \operatorname{sn}^{2 \nu} u\right) \operatorname{sn} u
$$

and

$$
\frac{d^{2 \nu+1} \operatorname{sn} u}{d u^{2 \nu+1}}=\left(a_{0}^{(\nu)}+3 a_{1}^{(\nu)} \operatorname{sn}^{2} u+\ldots+(2 \nu+1) a_{\nu}^{(\nu)} \operatorname{sn}^{2 \nu} u\right) \operatorname{cn} u \operatorname{dn} u
$$

where the $a^{(\nu)}$ are easily found by recursion which shows that they are integer functions of the squared modulus, $k^{2}$. Then, using the values sn $0=0, \operatorname{cn} 0=1$ and $\operatorname{dn} 0=1$, the Taylor series of $\operatorname{sn} u$ follows directly. A similar technique holds for $\mathrm{cn}$ and $\mathrm{dn}$ and for the other elliptic function combinations. Some coefficients are given in [19] IV pp.92,94.

Obviously one can just use the series for the three basic functions, sn, cn and $\mathrm{dn}$, to construct those for the others, say for $\mathrm{ds}=\mathrm{dn} / \mathrm{sn}$, by further recursion or inversion. One could also proceed directly, as for sn, using $\mathrm{ds}^{\prime 2}=\left(k^{2}+\mathrm{ds}^{2}\right)\left(\mathrm{ds}^{2}-\right.$ $\left.k^{\prime 2}\right)$.

A related technique, and the one referred to in the main body of this paper, is to use the derivative relations (we give these for the case we are interested in)

$$
\frac{d}{d u} \operatorname{ds} u=-\operatorname{cs} u \cdot \operatorname{ns} u, \quad \frac{d}{d u} \operatorname{cs} u=-\operatorname{ns} u \cdot \operatorname{ds} u, \quad \frac{d}{d u} \operatorname{ns} u=-\operatorname{ds} u \cdot \operatorname{cs} u ，
$$


mutually connecting the trio of functions, ds, ns, cs.

The conventional way of extracting the information in these relations consists of setting up three coupled recursion relations for the expansion coefficients of the three functions, e.g. Fricke [52], p.400. Alternatively, further differentiation yields the differential equation for ds,

$$
d s^{\prime \prime} u=2 d s^{3} u-\left(1-2 k^{2}\right) \mathrm{ds} u,
$$

and substitution of the expansion (32) yields, after a little algebra, the cubic recursion for $j \geq 3$,

$$
\begin{aligned}
\eta_{j}=\frac{4(2 j-1) !}{(j-2)(2 j+1)} \sum_{l=1}^{j-2} & {\left[\frac{3 \eta_{l} \eta_{j-l}}{(2 l-1) !(2 j-2 l-1) !}+\right.} \\
& \left.\sum_{n=1}^{j-l-1} \frac{4 \eta_{l} \eta_{n} \eta_{j-l-n}}{(2 l-1) !(2 n-1) !(2 j-2 l-2 n-1) !}\right] .
\end{aligned}
$$

This is the fermion analogue of the boson recursion, (14), coming from $\wp$.

In content, (67) is the same as the three coupled recursions, but is more compact and clearly shows that all coefficients are determined by just the first two, $\eta_{1}$ and $\eta_{2}$. It is quickly checked that the results agree with those listed by Glaisher and leads to (43) and (49). It may not be the most efficient way of determining the highest polynomials, but it is formally instructive.

For completeness we mention the second approach, adopted by Weierstrass, $[54,55]$, who writes, following Abel, the elliptic functions as ratios of power series by representing them as ratios, effectively of theta functions but more handily of the earlier Abel functions, $A l_{i}(u)$, which are just factors different from theta functions.

One has

$$
\operatorname{sn} u=\frac{A l_{1}(u)}{A l_{0}(u)}, \quad \operatorname{cn} u=\frac{A l_{2}(u)}{A l_{0}(u)}, \quad \operatorname{dn} u=\frac{A l_{3}(u)}{A l_{0}(u)}
$$

Weierstrass' next step is to Taylor expand the $A l_{i}(u)$ in a double power series in $k^{2}$ and $u$ using the differential equations that they satisfy. The coefficients are then determined by recursion. The calculation is straightforward and well adapted to machine evaluation. The result is the series,

$$
\begin{aligned}
& A l_{0}(u)=\sum_{m=0}^{\infty}(-1)^{m-1} C_{m} \frac{u^{2 m}}{(2 m) !}, \quad A l_{1}(u)=\sum_{m=0}^{\infty}(-1)^{m} A_{m} \frac{u^{2 m+1}}{(2 m+1) !} \\
& A l_{2}(u)=\sum_{m=0}^{\infty}(-1)^{m} B_{m} \frac{u^{2 m}}{(2 m) !}, \quad A l_{3}(u)=\sum_{m=0}^{\infty}(-1)^{m} D_{m} \frac{u^{2 m}}{(2 m) !},
\end{aligned}
$$


with $A_{0}=B_{0}=D_{0}=1$ and $C_{0}=-1, C_{1}=0$, from which the expansions for any elliptic function can be found, after a further recursion in the form of a forward substitution. For example, writing, cf (32),

$$
\operatorname{ds} u=\frac{A l_{3}(u)}{A l_{1}(u)}=\frac{1}{u}+\sum_{n=1}^{\infty}(-1)^{n} d_{n} \frac{u^{2 n-1}}{(2 n) !}
$$

one finds the relation between coefficients,

$$
\sum_{i=0}^{r-1}\left(\begin{array}{c}
2 r+1 \\
2 i+1
\end{array}\right) d_{r-i} A_{i}=(2 r+1) D_{r}-A_{r}
$$

and so

$$
(2 r+1) d_{r}=(2 r+1) D_{r}-A_{r}-\sum_{i=1}^{r-1}\left(\begin{array}{c}
2 r+1 \\
2 i+1
\end{array}\right) d_{r-i} A_{i}
$$

which is suitable for successive substitution. It is very easy to combine the recursions by machine, however Weierstrass [55] gives expressions for the $A_{m}, B_{m}, C_{m}, D_{m}$ coefficients up to $m=10$ and it is a simple matter to check that (70) reproduces the results from $(67)$.

This is an adequate scheme for practical purposes but somewhat utilitarian. An aesthetically more pleasing way of deriving the necessary series expansions is to use the equation for the twisted $\wp$-function, (56). In order to write this in terms of the modulus $k$, which corresponds to the original periods $\omega_{1}, \omega_{2}$, the modulus, $\widetilde{k}$, associated with the rotated periods, $\widetilde{\omega}_{1}, \widetilde{\omega}_{2}$, has to be related to $k$, with a corresponding statement holding for the complete functions, $K$ and $\widetilde{K}$. The transformation from $\omega_{1}, \omega_{2}$ to $\widetilde{\omega}_{1}, \widetilde{\omega}_{2}$ is a combination of modular transformations with a Gauss quadratic one, $c f$ [19] II,p.123, but a direct way of finding the relation is the following.

Standard relations in elliptic function theory are

$$
k=\sqrt{\frac{e_{2}-e_{3}}{e_{1}-e_{3}}}, \quad k^{\prime}=\sqrt{\frac{e_{1}-e_{2}}{e_{1}-e_{3}}}, \quad \frac{2 K}{\omega_{1}}=\sqrt{e_{1}-e_{3}},
$$

where the half-period values are,

$$
e_{1}=\wp\left(\frac{\omega_{1}}{2} ; \omega_{1}, \omega_{2}\right) \quad e_{2}=\wp\left(\frac{\omega_{1}+\omega_{2}}{2} ; \omega_{1}, \omega_{2}\right) \quad e_{3}=\wp\left(\frac{\omega_{2}}{2} ; \omega_{1}, \omega_{2}\right) .
$$

and so the problem is reduced to finding the relation between the $e_{\alpha}$ and the $\widetilde{e}_{\alpha}$. This can be done from the connection (53) by substituting in various values of $u$ and using the definitions (71). One has immediately that the last term in (53) is 
just $\widetilde{e}_{2}$. Putting $u$ equal to $\omega_{1} / 2, \omega_{2} / 2$ and $\omega_{3} / 2$ in turn in (53), in the first two cases the $\wp$ functions on the right are evaluated at quarter periods. Using standard results, e.g. [20] p.54, one quickly finds,

$$
\begin{aligned}
& e_{1}=\widetilde{e}_{2}-2 i \sqrt{\widetilde{e}_{2}-\widetilde{e}_{3}} \sqrt{\widetilde{e}_{1}-\widetilde{e}_{2}} \\
& e_{3}=\widetilde{e}_{2}+2 i \sqrt{\widetilde{e}_{2}-\widetilde{e}_{3}} \sqrt{\widetilde{e}_{1}-\widetilde{e}_{2}} \\
& e_{2}=\widetilde{e}_{1}+\widetilde{e}_{3}-\widetilde{e}_{2}=-2 \widetilde{e}_{2},
\end{aligned}
$$

and it is then easy to show that the moduli and complete integrals are related by

$$
2 \widetilde{k} \widetilde{k}^{\prime}=\frac{1}{2 k k^{\prime}}, \quad \widetilde{\lambda}=i k k^{\prime} \lambda
$$

It is to be remarked that the new modulus, $\widetilde{k}$, is complex and that $\widetilde{k}^{\prime}=\widetilde{k}^{*}$ so $\widetilde{k}^{2}=1 / 2+i b$ with $b$ real.

It is straightforward to compute the fermion partial energies from (23) and (57). According to (57) one needs the combination $2 \widetilde{g}_{2}-g_{2}$ which, after using $(73)$, gives,

$$
2 \widetilde{g}_{2}-g_{2}=-\frac{\lambda^{2}}{6}\left(8\left(k k^{\prime}\right)^{2}+7\right) .
$$

This is the same as in (49), up to a factor. To deal with $g_{3}$ we write it in the form given by Ramanujan [23] eqn.(60),

$$
g_{3}=\frac{8}{27} \lambda^{3}\left(1+\frac{1}{2}\left(k k^{\prime}\right)^{2}\right) \sqrt{1-\left(2 k k^{\prime}\right)^{2}} .
$$

This enables the transformations (73) to be simply applied in order to evaluate $2 \widetilde{g}_{3}-g_{3}$ which again reproduces the expression in (49). Note the useful symmetry

$$
\frac{i}{2 \widetilde{k} \widetilde{k}^{\prime}} \sqrt{1-\left(2 \widetilde{k} \widetilde{k}^{\prime}\right)^{2}}=\sqrt{1-\left(2 k k^{\prime}\right)^{2}} .
$$

From their structure, the quantities $\epsilon_{t}$ and $\eta_{t}$ will contain a polynomial in $2 k k^{\prime}$ for $t$ even and a similar polynomial times $\sqrt{1-\left(2 k k^{\prime}\right)^{2}}$ for $t$ odd. This is actually very handy when evaluating at singular moduli because the combination, $2 k k^{\prime}$, is often the most convenient one to emerge from the modular equation, e.g. [33].

In terms of the expansion coefficients in (68), $T$ inversion, (33), implies (compare with the scalar case, (48)),

$$
d_{n}\left(k^{2}\right)=(-1)^{n} d_{n}\left(1-k^{2}\right),
$$


and so a more appropriate variable would be $1 / 2-k^{2} \equiv \kappa$. For odd $n, d_{n}$ vanishes only at $\kappa=0$ while if $n$ is even, $d_{n}$ has a minimum at $\kappa=0$, maxima at $\kappa= \pm \kappa_{0}$ and passes through zero at $\kappa= \pm \kappa_{1}$, where $\kappa_{1} \rightarrow 1.5$ as $n \rightarrow \infty$.

Incidentally, purely as a check with Glaisher, using the modular transformations generated by $T: k \rightarrow k^{\prime}$ and $S: k^{\prime} \rightarrow 1 / k^{\prime}$, the expansion coefficients for the functions cs and ns are given in terms of the $d_{n}$. Those for cs are $k^{2 n} d_{n}\left(1 / k^{2}\right)$ and those for ns, $k^{2 n} d_{n}\left(-k^{2} / k^{2}\right)$. This means that the $d_{n}$ coefficients could also have been obtained by inverting the series for sn.

Gudermann (Weierstrass' teacher) in 1839 produced expansions which used the transformation relations for the elliptic functions (see [27]). Hermite devised a related technique which is outlined in Briot and Bouquet [45], see also [19] II p.209. One should also mention the work of André, [56]. More recently, a combinatorial formulation of the coefficients has been employed (e.g. Dumont [57]) which is more efficient for the highest coefficients. 


\section{Appendix C. Internal energies.}

In this appendix we display the remaining simpler singular case computations of the internal energies in three and five dimenions for scalars and spinors. This will give an indication of the complexity encountered, but the results are, at least, in finite terms.

First for scalars, in three dimensions,

$$
\begin{aligned}
\bar{E}_{3}\left(\xi_{6}\right) & =\frac{(35-20 \sqrt{2}+16 \sqrt{3}-14 \sqrt{6}) \Gamma^{4}(1 / 24)}{138240 \pi^{2} \Gamma^{4}(13 / 24)} \\
\bar{E}_{3}\left(\xi_{7}\right) & =\frac{17 \Gamma^{4}(1 / 7) \Gamma^{4}(2 / 7) \Gamma^{4}(4 / 7)}{458752 \pi^{8}} \\
\bar{E}_{3}\left(\xi_{10}\right) & =\frac{4(7725-5460 \sqrt{2}+3452 \sqrt{5}-2442 \sqrt{10}) \Gamma^{4}(9 / 40) \Gamma^{4}(7 / 8) \Gamma^{4}(41 / 40)}{\pi^{2} \Gamma^{4}(3 / 8) \Gamma^{4}(21 / 40) \Gamma^{4}(29 / 40)} \\
\bar{E}_{3}\left(\xi_{15}\right) & =\frac{(245-104 \sqrt{5}) \Gamma^{4}(1 / 30) \Gamma^{8}(4 / 15)}{86400002^{3 / 5} \pi^{2} \Gamma^{4}(1 / 6) \Gamma^{4}(1 / 3) \Gamma^{4}(17 / 30)},
\end{aligned}
$$

and in five,

$$
\bar{E}_{5}\left(\xi_{7}\right)=-\frac{\left(3808 \pi^{4} \Gamma^{4}\left(\frac{1}{7}\right) \Gamma^{4}\left(\frac{2}{7}\right) \Gamma^{4}\left(\frac{4}{7}\right)+171 \Gamma^{6}\left(\frac{1}{7}\right) \Gamma^{6}\left(\frac{2}{7}\right) \Gamma^{6}\left(\frac{4}{7}\right)\right)}{1233125376 \pi^{12}}
$$

The corresponding spinor expressions are

$$
\begin{aligned}
\bar{E}_{1}^{\mathrm{f}}\left(\xi_{6}\right) & =-\frac{2(1+2 \sqrt{2}-2 \sqrt{3}) \Gamma^{2}\left(\frac{25}{24}\right)}{\pi \Gamma^{2}\left(\frac{13}{24}\right)} \\
\bar{E}_{1}^{\mathrm{f}}\left(\xi_{7}\right) & =-\frac{\left(\Gamma^{2}\left(\frac{1}{7}\right) \Gamma^{2}\left(\frac{2}{7}\right) \Gamma^{2}\left(\frac{4}{7}\right)\right)}{128 \pi^{4}} \\
\bar{E}_{1}^{\mathrm{f}}\left(\xi_{10}\right) & =-\frac{\left(32-23 \sqrt{2}+4 \sqrt{\frac{1273}{10}-90 \sqrt{2}}\right) \Gamma^{4}\left(\frac{1}{40}\right) \Gamma^{4}\left(\frac{9}{40}\right) \Gamma^{2}\left(\frac{3}{4}\right)}{1280 \pi^{2} \Gamma^{2}\left(\frac{1}{20}\right) \Gamma^{4}\left(\frac{3}{8}\right) \Gamma^{2}\left(\frac{9}{20}\right)} \\
\bar{E}_{1}^{\mathrm{f}}\left(\xi_{15}\right) & =-\frac{(4-\sqrt{5}) \Gamma^{2}\left(\frac{1}{30}\right) \Gamma^{4}\left(\frac{4}{15}\right)}{3602^{\frac{4}{5}} \sqrt{5} \pi \Gamma^{2}\left(\frac{1}{6}\right) \Gamma^{2}\left(\frac{1}{3}\right) \Gamma^{2}\left(\frac{17}{30}\right)}
\end{aligned}
$$

and

$$
\bar{E}_{3}^{\mathrm{f}}\left(\xi_{7}\right)=\frac{224 \pi^{4} \Gamma^{2}\left(\frac{1}{7}\right) \Gamma^{2}\left(\frac{2}{7}\right) \Gamma^{2}\left(\frac{4}{7}\right)+15 \Gamma^{4}\left(\frac{1}{7}\right) \Gamma^{4}\left(\frac{2}{7}\right) \Gamma^{4}\left(\frac{4}{7}\right)}{114688 \pi^{8}} .
$$




\section{References.}

1. Dowker,J.S. Zero modes, entropy bounds and partition functions. hep-th /0203026.

2. Camporesi,R. Phys. Rep. 196 (1990) 1.

3. Cardy,J.L. Nucl. Phys. B366 (1991) 403.

4. Dowker,J.S. and Kennedy,G. J. Phys. A11 (1978) 895.

5. Gibbons,G.W. Phys. Lett. 60A (1977) 385.

6. Gibbons,G.W. and Perry,M. Proc. Roy. Soc. A358 (1978) 467.

7. Dowker,J.S. Phys. Rev. D37 (1988) 558.

8. Dowker,J.S. Class. Quant. Grav. 1 (1984) 359.

9. Cahn,R.S. and Wolf,J.A. Comm.Mat.Helv. 51 (1976) 1.

10. Dowker,J.S. and Banach,R. J. Phys. A11 (1979) .

11. Hurwitz,A. Math. Ann. 18 (1881) 528.

12. Hurwitz,A. Mathematische Werke Vol.I. Basel, Birkhauser, 1932.

13. Rademacher,H. Topics in analytic number theory, Springer-Verlag, Berlin,1973.

14. Rao,M.B. and Ayyar,M.V. J. Indian. Math. Soc. 15 (1923/24) 150.

15. Malurkar,S.L. J.Ind.Math.Soc 16 (1925/26) 130.

16. Hardy,G.H. J. Lond. Math. Soc. 3 (1928) 238.

17. Berndt,B.C. Rocky Mountain J. Math. 7 (1977) 147.

18. Glaisher,J.W.L. Messenger of Math. 18 (1889) 1.

19. Tannery,J. and Molk,J. Fonctions Elliptiques, Gauthier-Villars, Paris, 18931902.

20. Halphen,G.-H. Traité des Fonctions Elliptiques, Vol 1, Gauthier-Villars, Paris, 1886.

21. Hurwitz,A. and Courant,C. Allgemeine Funktionentheorie, Springer, Berlin, 1922.

22. Schwarz,H.-A. Formeln und Lehrsätzen zum Gebrauche.., Springer 1893.(The first edition was 1885.) The French translation by Henri Padé is Formules et Propositions pour L'Emploi..., Gauthier-Villars, Paris, 1894

23. Ramanujan,S. Trans.Camb.Phil.Soc. 22 (1916) 159; Collected Papers, Cambridge, 1927

24. Berndt,B.C. J. f. reine u. Angew. Math. 303/304 (1978) 332.

25. Al'taie, M.B. and Dowker, J.S. Phys. Rev. D18 (1978) 3557.

26. Whittaker,E.T. and Watson,G.N. Modern analysis, Cambridge 1927.

27. Hancock,H. Theory of elliptic functions. Vol I. Wiley, New York 1910. 
28. Watson,G.N. J. Lond. Math. Soc. 3 (1928) 216.

29. Schlömilch,O. Ber. Verh. K. Sachs. Gesell. Wiss. Leipzig 29 (1877) 101-105; Compendium der höheren Analysis, Bd.II, 3rd Edn, Vieweg, Brunswick, 1878.

30. Weber,H.M. Lehrbuch der Algebra Bd.III, Vieweg, Brunswick 1903.

31. Greenhill,A,G. The Applications of Elliptic Functions, MacMillan, London, 1892.

32. Appell,P. and Lacour,E. Fonctions Elliptiques, Gauthier-Villars, Paris, 1897.

33. Russell,R. Proc. Lond. Math. Soc. 19 (1888) 91.

34. Weber,H.M. Elliptische Funktionen und algebraische Zahlen, Vieweg, Brunswick 1891.

35. Greenhill,A.G. Proc. Lond. Math. Soc. 19 (1888) 301.

36. Berndt,B.C. and Chan,H.H. Mathematika 42 (1995) 278.

37. Glasser, M.L. and Wood,V.E. Maths of Comp. 25 (1971) 535.

38. Selberg,A. and Chowla,S. J. f. reine u. Angew. Math. 227 (1967) 86.

39. Zucker,I.J. Math.Proc.Camb.Phil.Soc 82 (1977) 111.

40. Zucker,I.J. Siam J.Math.Anal. 10 (1979) 192,

41. Zucker,I.J. and Robertson,M.M. Math.Proc.Camb.Phil.Soc 95 (1984) 5.

42. Joyce,G.S. and Zucker,I.J. Math.Proc.Camb.Phil.Soc 109 (1991) 257.

43. Zucker,I.J. and Joyce.G.S. Math.Proc.Camb.Phil.Soc 131 (2001) 309.

44. Borwein,J.M. and Zucker,I.J. IMA J.Math.Anal. 12 (1992) 519.

45. Borwein,J.M. and Borwein,P.B. Pi and the AGM, Wiley, New York, 1998.

46. Kutasov,D. and Larsen,F. JHEP 0101 (2001) 1.

47. Oshima,K. Phys. Rev. D46 (1992) 4765.

48. Cox,D.A. Primes of the form $x^{2}+n y^{2}$, Wiley, New York, 1989.

49. Kennedy,G. Phys. Rev. D23 (1981) 2884.

50. Dowker,J.S. and Critchley,R. Phys. Rev. D15 (1976) 1484.

51. Kiyek,K. and Schmidt,H. Arch.Math. 18 (1967) 438.

52. Fricke,R. Die Elliptische Funktionen und Ihre Anwendungen, Teubner, Leipzig. 1915, 1922.

53. Königsberger,L. Vorlesungen über die Theorie der Elliptischen Funktionen, Teubner, Leipzig, 1874.

54. Weierstrass,K. J.f.Mathematik (Crelle) 52 (1856) 346.

55. Weierstrass,K. Mathematische Werke Vol.I,p.1, Mayer u. Müller, Berlin, 1894.

56. André,D. Ann.École Normale Superior 6 (1877) 265; J.Math.Pures et Appl. 5 (1878) 31.

57. Dumont,D. Adv. in Math. 41 (1981) 1. 